\title{
Rising Data Availability in Cloud Using Graining Concept
}

\author{
Shivamma, R. Ramya, K. Chaithra and B. Raghavendra Rao
}

\begin{abstract}
Cloud computing is a technology with the intention of uses the internet and essential remote servers to maintain data and applications. Cloud computing allows clients and businesses to use applications without installation and admission their individual documentation at any computer with internet access. This equipment allows for much more efficient computing by centralizing storage, memory, processing and bandwidth. Cloud computing provides computation, software applications, data access, data administration and cargo space resources lacking requiring cloud users to know the position and added details of the computing transportation In cloud data are stored in cloud attendant in continuous format. When further number of users try to access same file then traffic will come about in cloud. To solve this trouble we planned mounting Data accessibility in Cloud using Seeding Concept (IDACC) model in cloud computing which helps to reduce interchange in cloud and cost of the cargo space data.
\end{abstract}

Keywords--- Cloud Computing, Data Availability, Security, Storage, Cost-Effective, Cloud Service Provider.

\section{INTRODUCTION}

$\mathrm{T}$ HE end of this decade is obvious by a paradigm shift of the industrial in sequence technology towards a payment based or pay-per-use service business reproduction known as cloud computing. This model provides users with a long list of advantages, such as terms computing capabilities; expansive various network accesses; resource pooling and rapid elasticity with measured services. Huge amount of data being retrieved from geographically scattered data sources, and non-contained data - behavior supplies, creates such a change in technical in addition to business model. One of the famous services offered in cloud computing is the cloud data storage space, have to pay the service providers for this storage service.

This service does not only provides elasticity and scalability for the data storage, it also make available regulars with the benefit of paying only for the amount of data they need to store for a exacting period of time, without any concerns for efficient storage mechanisms and maintainability with big amounts of data storage.

Shivamma, Student, Dept. of CSE, SSCE, Banglore, India. E-mail:sheeba123456789@gmail.com

R. Ramya, Student, Dept. of CSE, SSCE, Banglore, India. E-mail:ramyarnaidu1998@gmail.com

K. Chaithra, Student, Dept. of CSE, SSCE, Banglore, India. E-mail:chaithrak78@gmail.com

B. Raghavendra Rao, Asst. professor, Dept. of CSE, SSCE, Bangalore, India. E-mail:rhaghavendraraob.cse@sairam.edu.in

DOI:10.9756/BIJSESC.8282

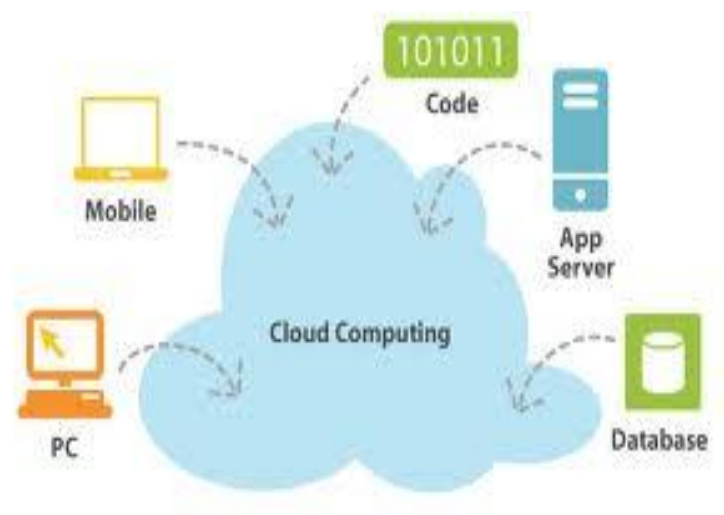

Figure 1: Cloud Computing

Among several SPs existing in the market, based on his existing financial plan. Also we provide a In adding to these benefits, customer's container easily contact their data from any geographical section where the Cloud Service Provider's network or Internet can be accessed. Along with these ented unpreced compensation, cloud data storage as well redefines the safety measures issues under attack on customer's outsourced data (data that is not stored / retrieved from the costumers own servers).Since cloud service providers (SP) are divide marketplace entities, data honesty and seclusion are the most serious issues that require to be addressed in cloud computing. smooth though the cloud service providers have typical policy and dominant transportation to make sure customer's data space to yourself and make available a better accessibility, the news of space to yourself break and examination outage have been clear in last a small number of years and. Also the political control might become an issue with the accessibility of services.

In this work we experimental that, from a customer's point of view, relying upon a solo SP for his outsourced data is not very gifted. In addition, provided that better time alone in addition to make sure data accessibility, can be achieved by separating the user's data building block into data pieces and distribute them among the accessible SPs in such a way that no less than a entry number of SPs can take part in victorious recovery of the whole data building block. To speak to these issues, hence less than a threshold digit of SPs can take part in winning recovery of the whole data chunk to address these issues in this paper; we planned an economical allocation of data between the existing SPs in the market, to provide clients with data availability as well as safe storage. In our model, the client divides his data decision for the client to which SPs he must chose to contact data, with value to data access feature of 5. 


\section{RELATED WORK}

Privacy safeguard and data openness are two of the most critical safety issues connected to user data. inside conservative model the organizations had the physical control of their data and hence have a case of implement improved data security policies. But in case of cloud computing, the data is stored on an self-regulating business gather that provides data storage as a payment service. The users have to trust the cloud service dealer (SP) with safety of their data. In, the writer discussed the criticality of the privacy issues in cloud computing, and sharp out that obtaining in order from a third party is much easier than from the creator himself. Following the pattern of model shift, the security policies also evolved from the conventional cryptographic schemes practical in central and discrete data storage, for enable the data privacy. Many of the cryptographic approaches have been designed for hitting the data from the storage space donor and preserving data privacy. In this authors outlook a scheme in which, the user's independence is also separate from the data, and claim to provide public auditing of data.

These approaches contemplate on one single cloud service provider that can easily become a blockage for such services. In, the authors studied and proved that sole cryptographic events are insufficient for ensuring data privacy in cloud computing. They also argued that the security in cloud storage needs a hybrid model of privacy enforcement, distributed computing and complex trust ecosystems. One bigger concern that arises in such method of cloud storage services is that, there is no full-proof way to be sure that the overhaul provider does not retain the user data, even after the user opts out of the payment. With huge amount of time, such data can be decrypted and significant information can be retrieved and user retreat can easily be breached. Since, the user might not be availing the storage services from that service supplier he will have no clue of such a inactive attack. The improved the cryptographic scheme, the more complex will be it is completion and hence the service provider will Data pieces connecting more than one service providers, in such a way that no one of the SPs can improve various important information from the pieces of data stored on its servers, without getting different more pieces of data from other service providers. Therefore, the directly single service provider based cryptographic techniques does not look too much talented .In, the authors discuss distributing the data over multiple clouds or network in such a way that if an participant is able to interrupt in one network, silent he cannot retrieve any important data since its equivalent pieces are stored in the other network.

Our advance is like to this move near, because both aim to remove the centralized separation of cloud data even though, in their approach, if the competitor causes a service outage even in one of the data networks, the user data cannot be retrieve at all. This is why in our model, we propose to use a needless allotment scheme, such as in, in which at least a threshold number of pieces of the data are necessary out of the whole distribution range, for successful retrieval.

\section{MODELS}

First in this segment, we will describe our system model and the danger model. Then, properly we will explain our problem speech we are going to study in this paper. Note that, in this work the terms cloud service source and service provider are interchangeable, the terms cloud storage and cloud data storage are compatible, also the terms user and client are interchangeable.

\section{A. System Overview}

We consider the storage services for cloud data storage among two entities, cloud users (U) and cloud check providers (SP). The cloud storage service is normally priced on two factors, how much data is to be stored on the cloud servers and for how long the data is to be stored. In our model, we assume that all the data is to be stored for same period of time. We consider $\mathrm{p}$ number of cloud service providers (SP), each available cloud service provider is associated with a QoS factor, along with its cost of providing storage service per unit of stored data (C). Every SP has a different level of quality of service $(\mathrm{QoS})$ offered as well as a different cost associated with it. Hence, the cloud user can store his data on more than one SPs according to the necessary level of security and their reasonable budgets.

\section{B. Warning Model}

Customers' stored data at cloud check provider is weak to various pressures. Previous studies in, discuss in detail that a cloud service provider can be a victim to rejection of service attacks or its variants. 626 In our work, we consider two types of warning model. First is the single point of failure, which will affect the data accessibility that could occur if a server at the cloud check source failed if not stopped, which makes it harder for the costumer to pull through his stored data from the server. Accessibility of data is also an significant issue which could be precious, if the cloud check provider (SP) runs out of business. Such worries are no more imaginary issues, consequently, a cloud service shopper can not fully rely upon a alone cloud service provider to make certain the cargo space of his essential data.

To show this risk we use an case in Fig. 2. Let us suppose that three patrons $(\mathrm{C} 1, \mathrm{C} 2$ and $\mathrm{C} 3)$ store their data on three dissimilar check parts (D1 and D2) and share out these parts on the two data into two presented CSPs (CSP1 and CSP2) in that order. The two cloud check provider is accessible for consumer (C1), who wants to store his own data strongly. In here he will separate his and restore the parts of data that the patron has store on their server and recreate the whole data not including being detected by the user on their server and rebuild the whole data without being detected by the user. Cloud service provider might plan with each other, providers (CSP1, CSP2 and CSP3) respectively. Each customer can get back his own data from the cloud service provider who it has a agreement with. If a breakdown occur at CSP1, due to inner difficulty with the head waiter or some issues with the cloud service contributor, all C1's data 


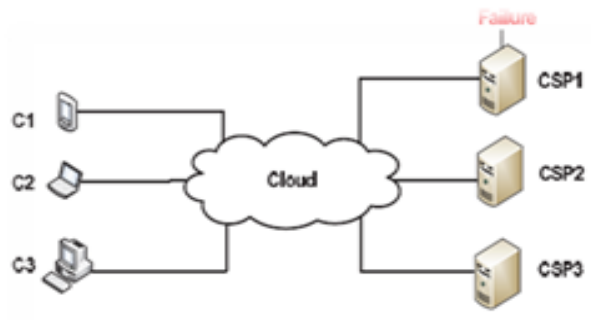

Figure 2: CSP Failure

Data accessibility in Cloud using Seeding impression (IDACC) which was stored on CSP1's servers will be lost and cannot be retrieved .One solution for this danger is that, the user will seek near store his data at several service providers to ensure better accessibility of his data. Our second threat discuss in this paper is the colluding service providers, in which the cloud service providers might collude mutually to reconstruct and admission the user stored data.

In this authors supply the idea for distributing the data among two storage space clouds such that, an challenger cannot get back the inside of the data without having access to both the storage break clouds.

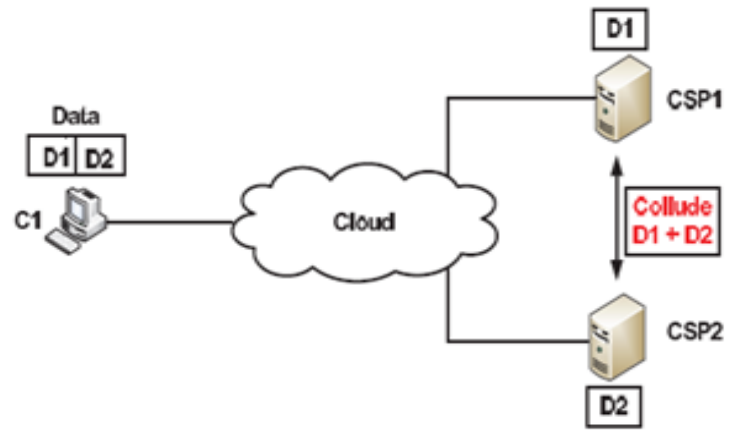

Figure 3:Colluding Cloud Service Providers

Relying totally in the lead a couple of services providers for the storage and loss of data might not be protected next to collude check providers. Such an bother situation is totally passive, since the cloud user cannot distinguish that his in order has been jointly retrieved from the check providers without his authority. We show the colluding check providers' threat in Fig. 3.

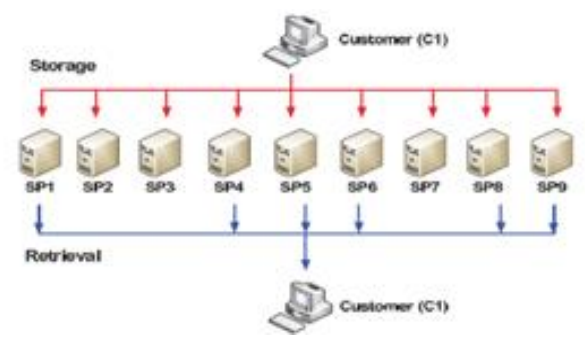

Figure 4: Data storage and Retrieval

\section{Problem Declaration}

In this part, we will properly state the explanation of our trouble that we are going to learn. Given $p$ number of cloud units (Ci). Our Increasing seeks a allocation of customer's data pieces among the available SPs in such a way that, at least q number of SPs must take part in data recovery, while minimizing the total cost of storing the data on SPs as well as maximizing the quality of service provided by the SPs.

\section{GROWING DATA ACCESSIBILITY IN CLOUd USING SEEDING CONCEPT}

In this work, to lessen the fear in front of cloud storage, we complete the cloud data storage to include compound service providers, where each cloud storage represent a dissimilar service provider. Our inspiration at the rear such an additional room is that, the adversary, similar to any other cloud user, is alert from the definite clouds of servers implemented by dissimilar cloud service supplier.

\section{CONCLUSION}

In this paper, we planned a growing Data accessibility in Cloud using Seeding Concept(IDACC)in cloud compute, which seeks to present each customer with a improved cloud data storage decision, taking into compassion the user plan as well as provide him with the best feature of check (Security and availability of data)accessible by accessible cloud service providers. By dividing among distributing customer's data, our model has shown its ability of providing a customer with a available storage under his affordable plan

\section{REFERENCES}

[1] P. Samarati and S. De Capitani Di Vimercati, "Data Protectionin Outsourcing Scenarios: Issues and Directions", Beijing, China, Pp. 1-14, 2010.

[2] W. Wang, Z. Li, R. Owens and B. Bhargava, "Secure and Efficient Access to Outsourced Data", In Proceedings of the ACM workshop on Cloud computing security, pp. 55-66, 2009.

[3] G. Aggarwal, M. Bawa, P. Ganesan, H. Garcia-Molina, K. Kenthapadi, R. Motwani and Y. Xu, "Two can keep a secret: A distributed architecture for secure database services", CIDR 2005.

[4] V. Ciriani, S.D.C. Di Vimercati, S. Foresti, S. Jajodia, S. Paraboschi and P. Samarati, "Keep a few: Outsourcing data while maintaining confidentiality", In European Symposium on Research in Computer Security, Pp. 440-455, 2009.

[5] V. Ciriani, S.D.C. Di Vimercati, S. Foresti, S. Jajodia, S. Paraboschi and P. Samarati, Keep a few: Outsourcing datawhile maintaining confidentiality, ESORICS, Saint Malo, France, 2009.

[6] V. Ciriani, S.D.C. Di Vimercati, S. Foresti, S. Jajodia, S. Paraboschi and P. Samarati, "Enforcing Confidentiality Constraints", 2009, Montreal, QC, Canada, 2009.

[7] V. Ciriani, S. De Capitani di Vimercati, S. Foresti, S. Jajodia, S. Paraboschi and P. Samarati, "Selective data outsourcing for enforcing privacy", Journal of Computer Security, Vol. 19, No. 3, Pp. 531-566, 2011. 